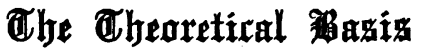

\author{
or
}

\section{THE SANOCRYSIN TREATMENT OF TUBERCULOSIS.}

\author{
BY
}

HOLGER MOELLGAARD, M.D.,

PROFESSOR OF PHYSIOLOGY, ROYAL VETERINARY AND AGRICULTURAL COLLEGE, COPENHAGEN.

Although chemical substances have been employed to a very large extent in the treatment of tuberculosis no real chemotherapy of this disease has hitherto been developed. The treatment here described represents an attempt to build up a chemotherapy in the sense of Ehrlich and Morgenroth; it cannot, however, be too strongly emphasized that this treatment is to be considered as a beginning only, and as probably showing a way out of the present embarras des richesses in the therapy of tubercúlosis.

Chemotherapy in the sense of Ehrlich is not to be understood merely as treatment with chemical substances only, and its effect is not due to any local action at the site of injection. Chemotherapy means the introduction into an infected organism of a substance having a directly injurious influence on the parasite and forcing this influence upon the parasite at long distances from the place of introduction. The possibility of such effect is intimately connected with a specific affinity of the substance for the parasite. Without such affinity it is hopeless to attempt to influence the parasites in any generally infected organism by means of chemical agents. As a specific affinity of any chemical substance to any parasite hardly exists without some affinity to the cells of the infected organism itself, the rate of parasitotropy to organotropy decides the question of the applicability of any substance as a chemotherapeutic agent. Ehrlich expressed this fact by means of the chemotherapeutical index. The existence of this index, however, means that a substance which is poisonous to a parasite will also exercise some toxic effect upon the infected organism. It is for science to reduce this toxic effect as much as possible, but it is necessary to emphasize the fact that even in the case of the most complete reduction of organotropy patients having a lesser tolerance to a certain compound than the average will be met with. Every injection of a chemical substance into the blood of any human being, therefore, always implies some risk, which must be balanced against the risk of giving no chemotherapeutic treatment.

To kill and dissolve bacilli in an organism by means of a bactericidal substance very often has the same effect as to inject killed or avirulent cultures-that is, it has an immunizing effect. The injection of the bactericidal substance produces an "ictus immunicatorius" (Ehrlich). Hence chemotherapy not only means bactericidal treatment, but also indirect immunization with liberated antigen. It may, however, under certain conditions produce another indirect effect. If the antigen is very toxic or is liberated in very large amounts, or the capacity of the organism for building antibodies for one reason or another is diminished, the injection of the bactericidal substance produces a more or less grave toxaemia, increasing in some cases to a deadly effect. We are already acquainted with such cases in the chemotherapy of spirilloses, and present knowledge of tuberculosis is in reality sufficient to make us expect the same to happen in the chemotherapy of that disease also.

The natural reaction of the therapeutist to this is to diminish the dose of the bactericidal substance. It must, however, be clearly understood that the effect not only depends on the quantity of the dose, but also on the amount of easily influenceable bacilli in the organism; further experience of chemotherapy has shown us that minimal doses are not only without effect, but in some cases even stimulate the disease ("effectus contrarius"-Ehrlich). Reduction of the dose, therefore, does not always solve the question of toxaemia. Where this effect is prominent chemotherapeutic treatment can hardly be carried through without the help of an active or passive immunization of the infected organism against the liberated toxins. In these cases clinical treatment must be carried out as a combined chemo- and sero-therapeutical treatment.

These are the principal ideas of chemotherapy founded on the work of Ehrlich and built up on the numerous investigations following in the wake of salvarsan.

For the last twenty years numerous chemical substances have been tried against tuberculosis, and the investigations into the influence of chemicals upon the tubercle bacilli outside and inside the organism are legion, the substances used ranging from dyestuffs to organic and inorganic compounds of heavy metals. In recent times German workers have made extensive investigations into the effect of heavy metals on tuberculosis (Tinkler, v. Linden, Meissen, Strauss, Bruch and Glück, Feldt and Spies, Bethmann, Juncker, Mayer, Pekanowitsch, Kolle and Schlossberger, Görke and Töppich, and others), as have also certain French, English, and American investigators (Breton, Hollande and Gaté, White, de Wit, Wells, Long, Cathwell, Leard, and others).

There is not space here to record the details of these investigations, but as far as $I$ can see their general results may be expressed by saying that they have proved that many compounds of heavy metals have a more or less pronounced growth-preventing influence on tubercle bacilli in culture, and that some of these compounds when injected intravenously retard the death of small tuberculous animals (rabbits, guinea-pigs), making the tuberculous lesions develop into a more sclerotic type. No real curative effect has, however, been observed.

From the failure to produce such effect, as well as from the fact that most of the metallic compounds employed have been too poisonous to the organism to be used in bigger doses in man, a new idea arose, supported first by pure theoretical considerations, but later on by experimentally proved facts, especially in Denmark (Walbum). In 1917 Feldt set forth his idea that the influence of the various metallic compounds (of gold, mercury, copper, bismuth, etc.) on the tuberculous processes is the result of a pure catalytic effect stimulating the natural reactions of defence of the organism - that is, its immunizatory processes. As a catalyser acts in very small concentrations, the logical consequence of this idea was the introduction of minimal doses into the therapeutics of tuberculosis. Feldt accordingly reduced the doses of krysolgan to a few milligrams injected at long intervals.

From Feldt's published statement it appears, as far as I can see, that this idea at the beginning was an ingenious but purely theoretical speculation. In recent times the possibility of exercising a stimulating effect on the production of antibodies by intravenous injections of small doses of various metallic salts has been proved by Walbum, and the whole idea of getting a clinical effect in this way supported by experimental facts.

On the other hand, no such clinical effect has at present been observed from most of the employed metallic compounds. Krysolgan seems only to have a detoxicating effect in certain cases of pulmonary tuberculosis and to cause quick healing in some cases of localized tuberculosis. The effect of the catalyser might, however, depend on its concentration in the body, as pointed out by Walbum, and recent investigations by him give support to this idea. Possibly, therefore, better results may be secured with some concentrations than with others, and there may be hope of something coming from the rational study of the influence of variations of the concentrations of metallic ions in the infected organism.

On the other hand, two things should never be forgotten : first, that the introduction of the minimal dose into human therapeutics in reality makes the assertion of the inefficacy of any substance on any kind of disease very difficult, especially in the case of the more chronic diseases, such as tuberculosis; and secondly, that a catalyser only augments the velocity of reaction and never alters the direction of chemical processes. By introducing a catalyser into a chemical system, whether a simple or complex system such as the organism, we cannot control the final 
results of its reactions but only the velocity with which the possible results are obtained. All catalytic treatment therefore implies a profound knowledge of the pathological conditions of the individual organism at the moment of beginning treatment, and it does not seem probable that important progress in clinical treatment will be made in this way before the true nature of the immunization processes in the organism have been cleared up to a larger extent than they are at present.

For this reason $I$ have felt it to be more hopeful to follow the lines of real ehemotherapy drawn by Ehrlich and Morgenroth. In the special case of tuberculosis there is, however, one reason more. The results hitherto obtained in the chemical therapy of this disease are, it is true, not very encouraging; but, on the other hand, I have noted that in none of the numerous investigations of the different kinds of chemical therapy of tuberculosis has any attention been paid to some of the most prominent facts in the pathology of the disease-the specific resistance of the bacillus of Koch and the peculiar structure of the tuberculous tissue. I considered, therefore, that an attempt to develop a rational chemotherapy of tuberculosis with special regard to these two facts was still worth making.

\section{A. The Chemistry of Sanocrysin Treatment.}

It is a well known fact that the toxic effect of a heavy metal on the organism is exerted by the positively charged metallic ion. A chemotherapeutical compound of a heavy metal must therefore never introduce important amounts of free metallic ious into the organism. Hence it follows that the first step in diminishing the organotropy of any compounds of heavy metal is to enclose the metal in a real complex body, of which the stability is sufficient to keep the concentration of metallic ions in watery solution practically oqual to 0 . Further, it is evident that the complex body in which the metal is enclosed must not itself be poisonous to the organism, as is cyanogen. Finally, the whole complex must have such chemical qualities that the amount of it which does not enter into combination with the parasite is excreted unaltered, or, if decomposed, then without the forming of metallic ions or other bodies poisonous to the organism.

These are the fundamental conditions which make it possible to use a compound of a heavy metal as a chemotherapeutic agent without injuring the organism. To secure a bactericidal effect the substance needs further a specific affinity for the parasite in question. The general principle on which to find such an affinity, when no prominent properties of the parasite suggest a special method, is laid down by Ehrlich and Morgenroth in " the principle of chemical variation." In tuberculosis the whole question, however, is complicated by two pocularities in its pathology.

1. The specific resistance of the bacillus of Koch is due to its content of fatty substances, whose presence is the principal reason for the so-called acid-fastness of the bacillus and its resistance to antiformin and other generally employed disinfectants. A bactericidal effect of any compound of a heavy metal on tubercle bacilli is, therefore, not to be anticipated, except subject to the condition that the compound is capable of penetrating the fatty system and so carrying the metal into the body of the bacilli.

2. The peculiarity of the tuberculous tissue, which is of importance in chemotherapy, is its poverty in vessels. This means that a chemical compound must diffuse from the blood through the tissue to reach the tubercle bacilli, and the distance through which diffusion must take place grows longer as the tubercle increases in size and develops in caseification. Whether a chemical compound under these conditions reaches the bacilli or not is a question, not only of its more or less pronounced specific affinity, but also of the ratio of its diffusion velocity to the velocity with which it is decomposed in or excreted from the organism. The liope for chemotherapy in tuberculosis is therefore probably intimately connected with the possibility of finding a substance which diffuses very quickly through animal membranes, and which is sufficiently stable in the organism to be kept unaltered in the blood and the lymph for a relatively long time.
These last considerations led to the idea that if a substance is to influence tubercle bacilli in the organism, its bactericidal effect must be carried by a rapidly travelling ion of high stability. As recent investigations (Hamburger, Ege, Gürber, Bunge, Abderhalden) appear to show that negatively charged ions diffuse much more quickly through animal membranes than positive ions, it seemed probable that the ideal chemotherapeutic in tuberculosis would be a negatively charged ion. Hence the fundamental idea in the chemical theory of sanocrysin is to insert the heavy metal into a real complex, which is a rapidly travelling negatively charged ion with sufficient stability to remain unaltered in the body for a relatively long time. The heavy metal carried by the sanocrysin ion is gold, because all previous investigations showed that it exercises a greater influence on tubercle bacilli in culture than any other metal. This quality is, of course, only of importance as a guide. To act as a chemotherapeutic agent the complex ion must possess a specific affinity for the tubercle bacilli, and must be able to carry the gold through the lipoid system into their body.

So far I have found the sanocrysin ion to be the body which best fulfils this condition. The chemical constitution of sanocrysin is very probably to be written as follows:

\section{$\left(\mathrm{AuS} 2 \mathrm{O}_{3}\right) \underset{\mathrm{Na2} \mathrm{S}_{2} \mathrm{O}_{3}}{\mathrm{Na}}$}

In watery solution it dissociates according to the equation : $\mathrm{Au}\left(\mathrm{S}_{2} \mathrm{O}_{3}\right)_{2} \mathrm{Na} 3=\mathrm{AuS}_{2} \mathrm{O}_{3}+\mathrm{S}_{2} \mathrm{O}_{3}+3 \mathrm{Na}$

Its therapeutic agent is then the complex negatively charged ion $\mathrm{AuS}_{2} \mathrm{O}_{3}$. This ion has, according to my investigations, the following properties. It-

(1) Is easily soluble in water (1 $\mathrm{gr}$. in $2 \mathrm{c.cm}$. water).

(2) Is a rcul complex and of great stability. Au is not generated by the usually employed strong reducing agents $\mathrm{H}_{2} \mathrm{SO}_{\text {SO }} \mathrm{SnCl}_{2} \mathrm{H}_{2} \mathrm{O}$, KI do not affect the substance even at boiling temperature. Dilute hydrochloric acid does not affect it at ordinary temperature. $\mathrm{H}_{2} \mathrm{~S}$ dccomposes the ion yielding gold sulphate. Highly concentrated watery solutions decompose slowly. especially in daylight, developing a yellow colour. Diluted solutions are unaltered in about fourteen days, when kept in a dark

(3) Is rapidly diffusible. The diffusion velocity of $\mathrm{AuS}_{2} \mathrm{O}_{3}$ through animal membranes is about 75 per cent. of the diffusion relocity of I-.

(4) Remains in the body for four to six days after injection into the blood.

(5) Is partly dccomposed in the organism, evidently yiclding metallic gold, and partly cxcreted principally by the kidneys in complex condition. When the urine contains gold it is not found in ionogenic form. $\mathrm{H}_{2} \mathrm{O}_{3}$ in alkaline solution decomposes the substance at $100^{\circ} \mathrm{C}$. totally into gold and $\mathrm{Na}_{2} \mathrm{SO}$.

(6) Does not prccipitate proteins, especially not the proteins of calf or horse serum, at a temperature of $38^{\circ}$ to $40^{\circ} \mathrm{C}$.

(7) Penetrates the lipoid system of the tubercle bacilli in a rery short time, affecting the acid-fastness very gravely and carrying gold into the body of the bacilli in amounts which after a short ime can be shown by microchemical reaction.

(8) Prevents the growth of tuberele bacilli in culture. On the medium I used, and which contained no peptone, I found the growth-limiting concentration to be 1 in 100,000. This value may, however probably be altered on media of other composition. however, probably be altered on media of other composition. Especially it must be kept in mind that all media containing $\mathbf{H}_{2}$ s will decompose the sanocrysin and precipitate the gold as
gold sulphate and metallic gold. In such media the growthgold sulphate and metallic gold. In such media the growth-
preventing concentration will increase; the question of growthpreventing properties is, however, of very small importance in chemotherapy. Dioxydiaminoarsenobenzol does not kill spirilli in ritro and is yet one of our strongest chemotherapeutic agents.

\section{B. Pharmacology of Sanocrysin: Effects on the} Healthy Organism.

As has been pointed out above, every chemical substance which has an affinity for a parasite may bo expected to have some affinity for the cells of the organism. The organotropy of sanocrysin has not been cleared $u$ in all its details, but the principal questions have been so far investigated that we have sufficient material to decide whether we can take the responsibility of injecting it into human beings or not. We know at present the following facts from investigations on calves, rabbits, and monkeys.

Circulution.-The intravenous injection of sanocrysin in doses of from 1 to $6 \mathrm{cg}$. per kilo of body weight does not affect the rhythm or rate of the heart; the blood pressure remains unchanged.

Red Blood C'orpuscles and Haemoglobin.-Repeated intravenous -injections of sanocrysin in doses up to $6 \mathrm{cg}$. per kilo body weight have no injurious effect on the red blood corpuscles or their haemoglobin. 
Temperaturo.-Doses up to $4 \mathrm{gg}$. per kilo body weight do not affect the body temperature.

Body Weight.-Doses up to $4 \mathrm{cg}$. per kilo body weight do not influence the weight of the individual.

Liver.-Doses up to $6 \mathrm{cg}$. per kiTo body weight have never caused any apparent injury to the liver and jaundice has never been observed.

Kidneys.-Sanocrysin is excreted principally by the kidneys. If a first dose of $2 \mathrm{cg}$. per kilo body weight is given it may be followed by slight albuminuria, which disappears within two or three daye If, on the other hand, the first dose does not exceed 1 cg. per kilo body weight, no albuminaria is caused; and subsequent doses of Q cg. per kilo body weight are tolerated without causing any albuminuria. Generally the dose can be increased gradually up to $6 \mathrm{cg}$. without causing albuminuria. Thus the kidneys rapidly increase their tolerance to sanocrysin.

Intcstinal Tract.-The administration of $2 \mathrm{cg}$. per kilo body weight produces no apparent effect on the intestinal tract. In monkeys $7 \mathrm{cg}$. have been tolerated without such effect.

Ncrvous System.-Doses up to $8 \mathrm{cg}$. per kilo body weight do not cause any detectable disturbance of the nervous system in healthy animals.

From these investigations it appears that sanocrysin in doses of from 1 to $4 \mathrm{cg}$. per kilo of body weight has very little effect on the sound organism. The weak point in the pharmacology of the substance is its action on the kidneys. This action is, however, avoided when the initial dose does not exceed $1 \mathrm{cg}$. per kilo nor the maximum therapeutical dose $2 \mathrm{cg}$. per kilo. Most animals endure bigger doses, and clinical experience has shown that the same is true in a large number of human beings.

On the other hand, the effect on the kidneys seems to be quickly reparable. Even with doses up to $6 \mathrm{cg}$. the albuminuria disappeared in a few days, and we have never found the lesions of the kidneys to develop in calves into chronic nephritis-even after six months' observation.

\section{Immunobrologicar Principles.}

From the preliminary investigations of Mafucci, as well "as from researches by many different authors (Prudden and Hodenpyl, Strauss and Gamaleia, Calmette and Breton, Grancher and Ledoux-Lelard, Ussmann, Kostenich, Krompecher, Keller, Engelhardt, Baumgarten, and others), we know that killed tuberele bacilli can exercise a local as well as a general injurious effect on the healthy organism. The local effect consists in the formation of abscesses at the site of injection, while when the bacilli are injected into the blood miliary tubercles are produced in different organs. The general effects are emaciation, cachexia, and more or less pronounced intestinal disturbances.

These results lead us to expect that a chemical compound which kills tubercle bacilli in the organism will cause very serious toxaemia when injected into a tuberculous organism.

Further, the splendid investigations of Robert Koch showed that the healthy and the tuberculous organism react quite differently to the injection of killed tubercle bacilli or tuberculin. In the healthy animal the toxic effect is produced only by large doses of killed tubercle bacilli and takes a relatively long time to develop. Even very small doses of killed bacilli or tuberculin often, in tuberculous animals, cause very severe symptoms, which develop in a relatively short time, and are signs of an acute intoxication of the organism. These symptoms are well known to everyone who is acquainted with the so-called specific therapy of tuberculosis. It need only be emphasized here that we meet two different groups of symptoms according to the pathological condition of the organism. The first group is represented by the acute intoxication caused by injection of tuberculin into highly tuberculous guinea-pigs and other small animals in the very acute stage of the disease. As shown by Koch, this "tuberculin shock" generally kills the guinea-pig in six to thirty hours, with very pro nounced and very constant pathological changes in different organs.

The other group is represented by the "tuberculin reactions" caused by injection of tuberculin or killed bacilli into tuberculous animals with a more chronic type of the disease. The principal symptom of this group is a rise in temperature, but when large doses of tuberculin are injected other symptoms are produced-namely, pain in the limbs, cough, pronounced faintness, nausea, and often vomiting. In many cases an exanthem similar to the rashes of measles appears on the neck and chest. Occasionally a slight icterus has been observed.

The injection into a tuberealous organism of a chemical substance which kills tubercle bacilli must therefore be expected to cause more or less pronounced symptoms of intoxication. As a general reaction the tuberculin shock has only been produced in guinea-pigs. Other animals seem to be more resistant. The production of shock in a larger animal might, however, only be a question of the amount of toxins formed in the body. It should therefore be strongly emphasized that to inject a bactericidal substance into a tuberculous organism may involve all the risks of tuberculin shock. At any rate, we must expect to find "tuberculous reactions," often in very pronounced degree.

On the other hand, it is clear that a chemical substance which is not capable of producing tuberculin reactions cannot be expected, when injected into tubereulous organisms not immune from tuberculin, to have any injurious inftuence on the tubercle bacilli. As almost all the larger (tuberculous) animals react to tuberculin, except in the earliest and latest stages of the disease, a curative effect produced by chemotherapy must always be associated with big reactious, unless we are able to produce total immunity, either active or passive, before the injection of the bactericidal substance.

From these considerations it follows that a substance capable of killing tubercle bacilli in the organism must also produce very severe reactions in the tuberculous organism when injected in doses which have no effect on a healthy animal. Is that true for sanocrysin? It has been mentioned above that this substance, injected intravenously in doses of $2 \mathrm{cg}$. per kilogram of body weight has no effect on the temperature, the body weight, and the intestinal canal, and does not cause albuminuria when a previous dose of $1 \mathrm{cg}$. has accustomed the kidneys to excrete the substance.

Experimental researches and the clinical experiences have shown quite uniformly that the tuberculous and the healthy organism react quite differently to this substance. A quarter, and in some cases one-tenth, of the dose, which is without effect on the healthy animal, produces the most severe reactions in the tubereulons organism, and in the experimental work and in clinical observations we have encountered all the different symptoms belonging to the two groups of symptoms observed after the injection of tuberculin into tuberculous animals and human beings.

\section{Shock Produced by Sanocrysin.}

Very early in my experiments $I$ found that one-third of the sanocrysin dose which is tolerated by the healthy gninea-pig always produces very severe intoxication in the highly tuberculous guirea-pig, and usually kills the animal in sixteen to forty-eight hours. The pathological changes in the organs have always been absolutely identical with those found by Robert Koch in the tuberculous gainea-pig killed by tuberculin; in particular a large haemorrhagio zone was found surrounding the tuberculous lesions and spreading into the tissue around them.

In further experiments on highly tubercalous animals (goats, calves, monkeys) the same phenomena were observed. In monkeys especially, which are very sensitive to the intoxication, the shock could be made very significant; when the infection is set up by intraperitoneal injection with a small dose of human tubercle bacilli, monkeys develop a chronic tuberculous peritonitis, which does not cause death until after a period of about three months or more. If in the third week after the infection so small a dose as only $2 \mathrm{cg}$. of sanocrysin per kilogram of body weight were injected intramuscularly in one hind leg, a serosanguineous peritonitis develops in six to twenty-four hours, and the animal dies usually within forty-eight hours.

Goats, and calves more especially, though they are more resistant, yet very often die after only one injection of $2 \mathrm{cg}$. of sanocrysin per kilogram of body weight. In all cases pathological changes were found in the tuberculous organs of the same kind as in the tuberculous guinea-pigs. In calves, which ordinarily do not die until forty-eight 
hours after the injection of sanocrysin, the development of the shock was investigated in detail. Ordinarily it begins with albuminuria, which develops into a grave parenchymatous nephritis. Shortly after the appearance of the grave symptoms of nephritis (increasing albuminuria, cylindrical casts and blood corpuscles in the urine) acute myocarditis appears. The third and last stage in the intoxication in animals affected with pulmonary tuberculosis is an extensive oedema of the lungs, which increases until the animals die, with frothy fluid exuding from the mouth and nose. Shortly before death a very significant clinical symptom occurs-namely, a fall in temperature, usually of $2^{\circ}$ to $3^{\circ} \mathrm{C}$. In most cases the animal dies at the lowest point in the temperature curve; but in some cases it recovers temporarily, to die one or two days later.

\section{Serotherapy of Sanocrysin Shock.}

Investigation of thirty large tuberculous animals treated with sanocrysin pointed to the fact that the shock mainly occurred during two distinct periods-namely, during the first three weeks after the artificial infection, and during the last weeks before death. As it is well known that the measurable antibodies in the blood are very much diminished, if not totally absent, in these two periods, $I$ came to the conclusion that animals in the more chronic period might contain antibodies which were capable of protecting them from the shock. To test this I injected serum from a calf, affected with chronic tuberculosis for about three months, iritravenously into two tuberculous calves suffering from grave shock produced by a sanocrysin injection. The result was striking; these calves recovered clinically in a few hours, and the albuminuria disappeared in twenty-four hours. From this observation I concluder that it might be possible to immunize animals against shock.

As the development of the shock is rapid there will never be time to produce an active immunity in an acute case; the protection against the shock must be by means of a passive immunization-that is, by way of serotherapy. The truth of this idea has been shown by demonstration that it is possible to protect tuberculous animals against the shock and to cure the shock by means of a serum produced by immunization of healthy animals with killed tubercle bacilli.

This serum was first produced in calves by repeated injections of killed tubercle bacilli and tuberculin. At present it is made by injection of defatted, formalin-treated tubercle bacilli, according to Dreyer's principle. By means of this antigen the strength of the serum has been increased, and it has been possible to make it in horses.

With this serum forty tuberculous calves and goats, suffering from albuminuria after sanocrysin injection, have been treated on more than 120 occasions. In all cases where the infection has been reasonable the albuminuria has disappeared, in most cases very quickly, but in some more slowly, and the shock was totally prevented. Only in cases in which the injection has been very much too large (animals infected with more than $7 \mathrm{cg}$. of virulent tubercle bacilli injected intravenously) the serum has not always been able to prevent the shock. This suggests that study of the serum question may contribute very much to the efficiency of the treatment in the grave cases of very acute progressive disease.

The experiments on calves and monkeys, as well as clinical tests on human beings, have uniformly shown that the serum is capable of curing the shock, when injected intravenously in sufficient doses at the very beginning of the fall in temperature. If, however, the collapse has continued for any considerable time, the serum injection may be followed by temporary recovery, but death ensues from failure of the heart. In clinical treatment it should therefore always be the rule to inject. serum at the earliest appearance of the albuminuria, as it is the first sign of impending shock. It is, of course, true that albuminuria is not on every. occasion followed by shock, but it is not always possible to decide in which case this will happen and in which it will not. The clinician must be very careful on this point, and not omit the serum treatment in any case before he has acquired sufficient experience to be able to judge the condition of his patients during the sanocrysin treatment.

As far as I can see, all these investigations prove that sanocrysin in tuberculous animals and human beings can produce an acute intoxication, which does not occur when much larger.doses are injected into healthy animals, and that this intoxication can be prevented or cured by means of a serum made by immunization of healthy calves and horses with defatted formalin-treated tubercle bacilli. Consequently it is possible to confer upon tuberculous animals and human beings a specific immunity against the lethal effect of sanocrysin. This is probably the best proof of the bactericidal influence of this substance upon tubercle bacilli.

\section{Reactions of the Tuberculous Organism Immune to the Shock.}

As has been pointed out above, animals with a more chronic tuberculosis very often show a high immunity to shock, and the same is true with certain tuberculous human beings. These animals or patients tolerate the sanocrysin injection without getting any albuminuria or other signs of the shock.

The tuberculous organism, whether naturally immune or made immune by serum, gives very pronounced reactions to sanocrysin, but of another very different type. None of the features of shock appear, but we find instead all the symptoms belonging to the second group mentioned above -the "tuberculous reactions." The most obvious of them are the following: rise in temperature, exanthemata, loss in weight, faintness, and intestinal disturbances. Besides them focal reactions in the infected organs are observed with more or less regularity, as happens with tuberculin injections.

There is no space to consider here all the details of these reactions; they have been investigated to a very large extent by the clinicians, who have proved a striking similarity in all principal points between the experimental and the clinical observations. For details I must refer readers to the preliminary report on results from Danish clinics (Holger Moellgaard, Chemotherapy of Tuberculosis, Copenhagen, 1924), and to later publications.

Loss of Reactions during Treatment.

One important feature of all the reactions mentioned must be emphasized: they all disappear gradually as the clinical signs of the disease disappear. This fact is very interesting, because it is just what might be expected if sanocrysin is really able to cure tuberculous disease. To do this the substance must be able to transform the tuberculous organism from a condition in which it gives a severe reaction into a condition in which it gives no reaction to the substance itself, as is the case with the healthy animal. The fact that this transformation is actually observed brings us nearer to the idea of a real cure of the disease. The cured tuberculous animal tolerates sanocrysin without getting albuminuria, temperature reactions, loss in weight, exanthemata, focal reactions, or intestinal disturbances.

On the other hand, the pathological conditions in tuberculosis are so complicated that the loss of all reactions to sanocrysin is no decisive proof of a real cure. From the animal experiments we already know that the condition of failing reactions to sanocrysin means one of three things:

1. Sterilization until negative result of the guinea-pig test.

2. Total immunity with loss of Pirquet reaction, but without sterilization.

3. Simple failure of reactions because the rest of the bacilli are too protected by fibrous tissue or lime; in these cases the Pirquet reaction persists.

Whether there is a fourth possibility-that of a goldfastness of the bacilli-we do not know at present. If such a condition exists it seems, however, to be temporary only, but further investigations are urgently needed.

From clinical experience in man we know already the last two conditions. To prove the first is, for valid reasons, beyond the reach of clinical observation at this early stage of our observations. Personally I doubt whether it will ever be possible to prove it clinically. 
Before their total disappearance the reactions to sanocrysin are ordinarily delayed until two or three days after injection. The transitory stage between the disappearance of the clinical signs of tuberculosis in the lungs and the definite loss of reactions is marked by "late reactions." This phenomenon is very interesting, because its appearance is in close accordance with the power of sanocrysin to remain for four days in the organism and to diffuse through animal membranes. The late reactions may be explained on the assumption that most of the bacilli have been cleared out, and those remaining are better protected; the substance, therefore, takes longer to reach the bacilli by diffusion.

This observation has a very important bearing on clinical trials. In experiments the late reaction has been found only in the last stages of the cure. Where, however, the bacilli are well protected by fibrous tissue (a condition present in many cases of chronic tuberculosis met with clinically), such reactions are to be expected from the beginning of the treatment. Clinical experience has shown that "late reactions" two to four days after a sanocrysin injection very often occur.

For the clinician it is of great importance to know that even big reactions may occur as late as four days after one sanocrysin injection, because it tells him that if he injects a fresh dose within this interval he may bring upon his patient the risk of a cumulative effect of the liberated toxins. If there are no special indications which make a more intensive treatment necessary the interval between two injections of $2 \mathrm{cg}$. of sanocrysin per kilo of body weight should never be shorter than four days. For the same reason it is not right to inject a fresh dose of sanocrysin during the temperature reaction produced by a previous injection.

\section{Curative Results.}

The general results of the animal experiments hitherto made may be summarized as follows: The combined sanocrysin-serum treatment has saved the life of even very gravely infected goats, calves, and monkeys, and brought them into a condition of clinical healing. On the other hand, it appears evident from the experiments that a thorough sterilization of the affected organs is very difficult and probably very seldom secured in cases of grave infections. In four lighter cases and in one grave case I have been able to carry the cure through until the guinea-pig test was negative. In all other experiments the guinea-pig test has been positive, though the tuberculosis was reduced to a few calcified tubercles in the langs and sclerotic processes in the bronchial glands.

In considering the clinical bearing of these observations it must be strongly emphasized that the curative results in the animal experiments have been secured mostly in cases of exudative pneumonic type of tuberculosis of the lungs. I have chosen this type in order to aroid the well known variations of the chronic type, which are very disturbing in experimental work. But by choosing this type I have, strictly speaking, limited the proof of the curative effect of the sanocrysin to exudative pneumonic tuberculosis in the lungs. Further, the results of the guinea-pig test in my experiments suggest that the possibility of achieving sterilization depends to some extent on whether the disease has progressed as far as caseous destruction or not. In the cases where the tests have given negative results the postmorter examination showed no tubercles or only very few. In all cases where a larger amount of bigger tubereles were found, even though they were calcified, the guinea-pig test gave positive results.

It must therefore be expected that the best effect of the sanocrysin-serum treatment in the human subject will be obtained in cases of the exudative pneumonic type. Clinical experience seems already to show that this consideration comprises a true idea, the most striking clinical results having been secured in exudative cases. As, however, our present treatment, inclusive of artificial pneumothorax, fails to give definite results in very many of these cases, the attempt to found a chemotherapy on sanocrysin would not have been in vain, even if it should be limited to such cases.

On the other hand, the experiments have demonstrated that it is possible in a short time to cause typical produc- tive [sic] tuberculosis ${ }^{1}$ in its earliest stages to heal up with sclerosis and calcification, and clinical experience has shown that cases of acute extensive miliary tuberculosis of the lungs can be brought into a condition of clinical healing. Even though the effect is greatest in the exudative cases, there might be some good results in the more productive cases also. Besides, clinical tuberculosis, as is well known, very seldom provides a pure case of one type of affection. In most cases there will be found productive and exudative processes together, and the sanocrysin will then have a great mission in checking the exudative progressive processes.

As was to be expected, the extent and gravity of the tuberculous process set a certain limit for the success of the sanocrysin treatment In experiments on calves and goats this limit has been found with an initial infection exceeding $7 \mathrm{cg}$. tubercle bacilli per $100 \mathrm{~kg}$. live weight of the cultures I have employed. I have only been able to carry one case with an initial infection of $10 \mathrm{cg}$. through until clinical healing. In the other cases the cure was inhibited by a rapidly developing cachexia, when big doses of sanocrysin were employed, and in cases of small doses the process could not be stopped: there were too many bacilli. It must, however, be understood that this limit is not decisive but dependent on the virulence of the cultures employed, when the treatment begins at the same time after the date of infection.

Finally, animal experiments have shown that other diseases in the lungs (mixed infections) may render it very difficult to obtain a curative effect with sanocrysin because the other processes themselves progress or protect the tubercle bacilli against the substance by enclosing them in large sclerotic masses of destroyed tissue. This has been observed in goats gravely infected in both lungs with Strongylus capillaris as well as with tuberculosis.

\section{E. The Fate of the Tubercle Bacilli in the Tissue and the Alterations of the Tubfrculous Lesions.}

In three cases I have examined the lungs of calves after two to four weeks' sanocrysin-serum treatment. In all cases microscopical examination of preparations stained by the Ziehl-Neelsen method showed, particularly in small remaining miliary tubercles and their surroundings, numerous acid-fast granulations mixed with curled acidfast and grey non-acid-fast bacilli. Amongst them were often found some acid-fast bacilli with normal morphology, and, as a transitory stage between the normal bacilli and the free granules, chains of granulations in the tissue. The granules were almost all intracellular. Probably these phenomena are indicative of the way the bacilli are cleared out of the tissue under the treatment. At any rate, these alterations of the bacilli in the tissue are, in my opinion, very remarkable.

The influence of the treatment on the anatomical lesions in the tuberculous organs may, it will be understood, vary according to whether the disease has been checked before the development of caseous tubercles or not. Where the process has been treated in the pneumonic exudative stage the only residuals of the process found on microscopical examination seem to be obliterated capillaries and small vessels and some infiltrations of the alveolar walls, which, howerer, are also found in lungs of quite normal calves.

Where real miliary tubercles and bigger caseous tubercles have developed, we have found the same alterations as are ordinarily observed when the tuberculous process heals spontaneously-namely, sclerosis and calcification. The most interesting observation has been that miliary tubercles may be grown through with fibrous tissue and contain a stone-hard piece of lime after four and a half to ten weeks' treatment. Bigger caseous tubercles have often in a very short time (five weeks) become surrounded by thick fibrous walls, and more or less filled with pieces of lime.

These results seem to prove that the sanocrysin treatment really exercises such influence on the bacilli in the tissue and on the tuberculous lesions as might be expected from the clinical observations of its curative influence on the tuberculous processes. 